\title{
Impact of the Dihedral Angle of Switched Beam Antennas in Indoor Positioning based on RSSI
}

\author{
Stefano Maddio ${ }^{1}$, Marco Passafiume ${ }^{2}$, Alessandro Cidronali ${ }^{3}$, Gianfranco Manes ${ }^{4}$ \\ Dept. of Information Engineering, University of Florence, V.S. Marta, 3, I-50139, Florence, Italy \\ $\left\{{ }^{1}\right.$ stefano.maddio, ${ }^{2}$ marco.passafiume, ${ }^{3}$ alessandro.cidronali, ${ }^{4}$ gianfranco.manes $\} @$ unifi.it
}

\begin{abstract}
This paper presents an investigation about Switched Beam Antennas (SBA) for indoor positioning systems based on RSSI measurements. Given practical consideration on hardware costs, the device in exam is based on low-cost commercial components capable of standard WiFi connectivity at $2.45 \mathrm{GHz}$.

The SBA is the enabling technology for Beam Diversity Multiple Access of smart WiFi nodes. Upon the reception of radio messages from generic mobile devices, the node estimates the DoA of the incoming signals on the basis of a likelihood criterion driven by the expcedted beam diversity.

SBA design has great impact on the localization performance. In this paper an investigation around the SBA shape is presented, with particular emphasis on the effects of the dihedral angle of regular polyhedron-type SBA. Thanks to a model based on trustful electromagnetic simulations, some conclusions on the general design principles of the SBA's are drawn.
\end{abstract}

Index Terms-Indoor positioning system, Switched beam antenna (SBA), Direction of arrival (DoA), RSSI.

\section{INTRODUCTION}

The interest of the scientific community about the positioning systems in GPS-denied scenarios has recently grown, especially in the field of Wireless Sensor Network (WSN) [1]. A network of nodes capable of position awareness is able to independently determine the best modality to cooperate and communicate the data to the end user, with interesting consequence over a wide spectrum of unattended activities.

Many localization approaches has been investigated in recent years, based on a wide set of auxiliary signal parameters, such as time of arrival, time difference of arrival, network connectivity and Received Signal Strength Indicators, RSSI.

The latter in particular, is typically employed for range estimation based on channel propagation model, or for sceneanalysis/fingerprinting. Unfortunately, the complexity of the radio channel, and the typical $1 \mathrm{~dB}$ resolution, poses a serous limit on the extraction of sensed magnitudes from RSSI.

RSSI measurements can also be employed for Direction of Arrival (DoA) estimation. In this case the noise issue has reduced impact, and can be further reduced with suitable architecture [2], [3]. The key of DoA estimation with RSSI is the Beam Division Multiple Access, the channel access method based on signal reception using directive antennas. A Switched Beam Antenna (SBA) is a specialized radiator capable of a predetermined set of directional beams. Operating as spatial multiplexer SBA alternatively isolates the signal reception from specific areas.

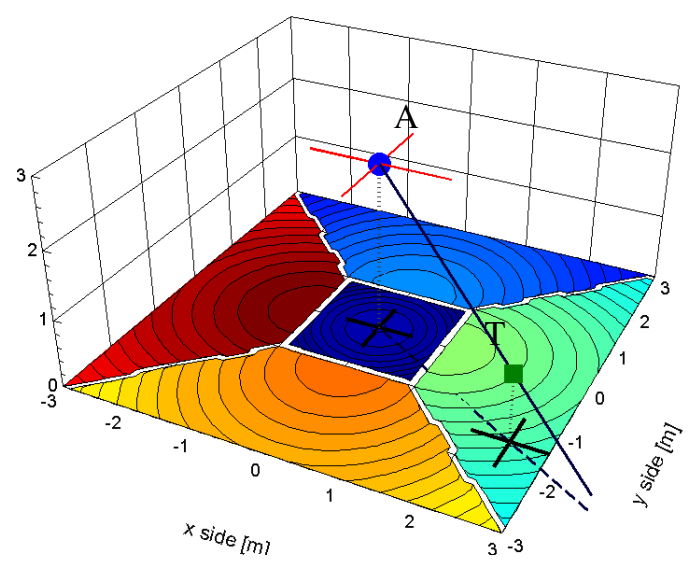

Fig. 1: Indoor localization based on a Switched Beam Antenna.

With reference to Fig. 1, a mobile node $T$ enters in the communication range of the specialized access point $A$. During the normal exchange of WiFi radio messages, $A$ estimates the DoA of the incoming messages comparing the RSSI with the expected set of antenna beams. The more discriminated is the beam set, the higher is the recognizability of the DoA. This angular position directly translates in a univocal positional information if the target quote is constrained.

In this paper, the effects of SBA arrangement is investigated. Chosen the antenna elements, the more impacting parameter is the dihedral angle of their arrangement. Section II describes the architecture of a SBA system, while Section III presents the localization strategy. Section IV presents the localization results due to various SBA arrangement and finally in Section $\mathrm{V}$ some conclusions has been drawn.

\section{ARCHITECTURE OF THE SYSTEM}

In the following, the architecture of a typical node operating with a SBA will be briefly summarized.

\section{A. Transceiver}

The core the device in exam is the class of modern integrated systems like the CC2430 from Texas Instruments. This system-on-a-chip comprises a sensible transceiver which is compliant with IEEE 802.15.4 and ZigBee, and comes with a built-in RSSI module which operates on signals averaged over an 8 symbol periods, returning a formatted data corresponding to the effective incoming power through a calibration equation. 


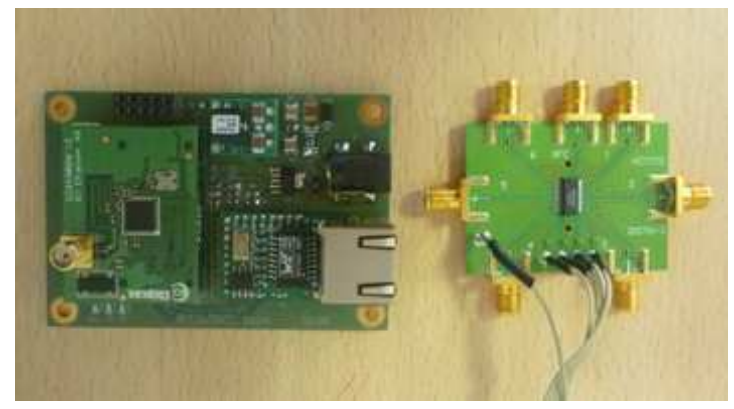

Fig. 2: Node mainboard and switch.

The beam diversity is activated by a Single Pole N Throw switch. Typically, GaAs SPNT presents insertion loss under $2 \mathrm{~dB}$ and an isolation around $30 \mathrm{~dB}$ at the nominal frequency of $2.45 \mathrm{GHz}$. With a control logic made of a three bit string, the switch is directly controlled the GI/O of the transceiver.

\section{B. Elementary antennas}

The elementary antennas are circularly polarized $(\mathrm{CP})$ patch realized in common planar technology on a cheap FR4 substrate. CP operation grants reliable link regardless of the relative orientation of antennas and it is an aid to contrast the multi-path impairment [4], making RSSI data much less noisy [2]. Fig. 3 depicts a prototype of compact CP antenna, called ESDA, designed on the basis of modal degeneration mechanism [5]. Capable of a directive symmetric CP beam the principal cut is illustrated in Fig. $3 b-$ this antenna was presented by the authors in a previous work [6], and it was already employed in indoor localization problem [3], [7].

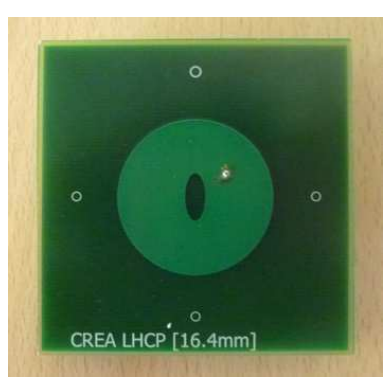

(a) Antenna Element

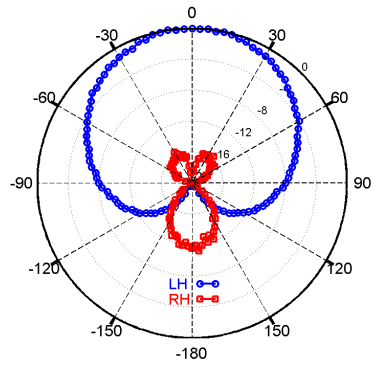

(b) Radiation Pattern
Fig. 3: Elementary antenna of the SBA.

\section{LOCALIZATION AlgORITHM}

The system in exam is designed to operate with likelihooddriven algorithms, such as MUSIC [8], [9], which estimates the signal DoA on the basis of the RSSI set measured by the SBA sectors. If the sequential reception is faster than the channel coherence time, no assumptions on the channel propagation model is necessary.

Assuming $S_{n}$ as the RSSI sampled by element n, the observation model is

$$
S_{n}=G_{n}(\theta, \phi)+P_{r x}+w_{n}
$$

where $G_{n}$ is the $n^{\text {th }}$ element gain (in $\mathrm{dB}$ ), $P_{r x}$ is the receiving power impinging on the SBA section and $w_{n}$ is an average white gaussian noise.

Let $\mathbf{S}(k)$ be the entire set of RSSI's sampled at time step k:

$$
\mathbf{S}[k]=\mathbf{S}\left(T_{0}+k \Delta t\right) .
$$

From $K$ repetition, the correlation matrix $R_{s s}$ of the received signals is estimated as [8]:

$$
\hat{R}_{s s}=E\left[\mathbf{S}[k] \mathbf{S}[k]^{\top}\right]=\sum_{k=i}^{K} \sigma_{m}^{2} \mathbf{G}\left(\theta_{s}, \phi_{s}\right) \mathbf{G}\left(\theta_{s}, \phi_{s}\right)^{\top}+\mathbf{I}
$$

where $\sigma_{m}^{2}$ is the SNR of the signal and $\left(\theta_{s}, \phi_{s}\right)$ is the real DoA. Thus, applying the single value decomposition:

$$
\left(R_{y y}\right)=U S U^{*}
$$

the space spanned by the involved signals can be partitioned as $U=\left[U_{s}, U_{n}\right]$, where the $N \times 1$ matrix $U_{S}$ is the signal subspace, and the matrix $U_{n}$ is the signal null space. Being $U$ an unitary matrix, the signal and noise subspace are orthogonal $\left(U_{S} U_{N}=1\right)$, therefore, a pseudo-spectrum, defined as

$$
\mathrm{P}_{\mathrm{S}}(\theta, \phi)=\frac{1}{\mathbf{G}(\theta, \phi) U_{n}}
$$

exhibits a maximum at the estimated DoA condition.

\section{IMPACT OF SBA CONFIGURATION}

SBA shape [10] and placement [11] have great impact in the performance of localization. In this section, the effects of SBA dihedral angle for the localization performance is analyzed. Throughout this paper the SBA-equipped node is intended hanging from the ceiling of an indoor area, and downfacing, with the $\theta=0$ angle corresponding to floor direction, at an height of $1.5 \mathrm{~m}$ respect to the floor reference. To simplify the analysis, the target is equipped with an identical CP antenna, facing upward.

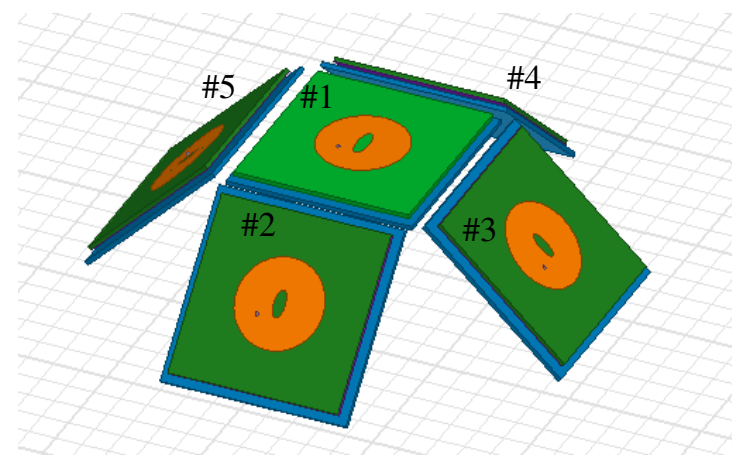

Fig. 4: 3D model of the 5-faced cubic-like SBA.

\section{A. Dihedral Angle of the SBA}

Considering that antenna pattern in Fig. $3 b$, to meet the requirement of uniformity, a regular solid is the most obvious choice. We limit the analysis to a cubic-based structure as the one depicted in Fig. 4. With reference to the figure, the top antenna element is labeled as \#1, while the side antenna 
elements are \#2-\#5. The dihedral angle is defined as $\alpha$ inside Fig. 6.

Arranged in this regular structure, each antenna tends to be at its maximum radiation where the other are in lowgain, a condition which helps to make angularly uncorrelated informations. At the same time the cumulative pattern, - i.e. the envelope of all the radio beams - can guarantee uniform reception over the entire area of interest.

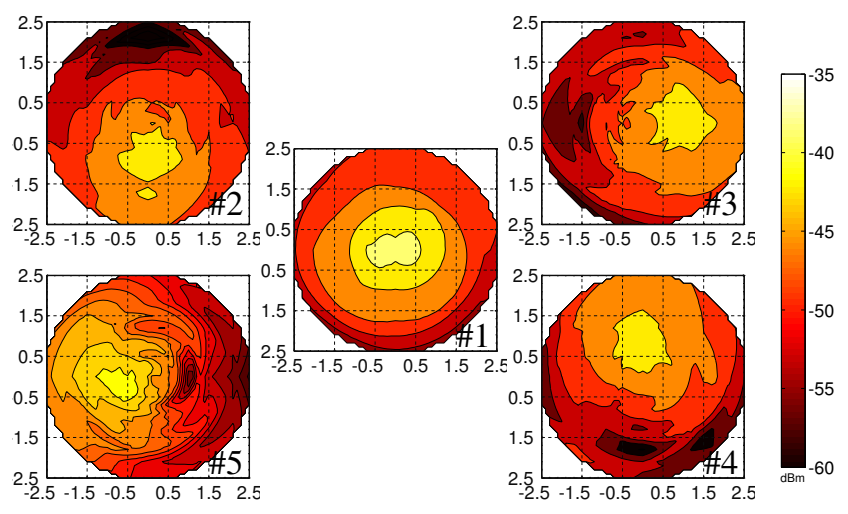

Fig. 5: Typical distribution of RSSI revealed by the five antennas for the case of dihedral angle $=110^{\circ}$.

By the mean of a full-wave electromagnetic simulation, the power signal distribution of an indoor link is estimated as in Fig. 5. The subplots depict the sensed RSSI revealed by each antenna within a circular range of 2.5 meters. The 5 peaks, one for each elements, identify five areas as qualitatively described in Fig. 1. This space partition is effective for the creation of a localization cell, which grants an uniform tiling of the indoor area if a set of anchors is displaced [3].

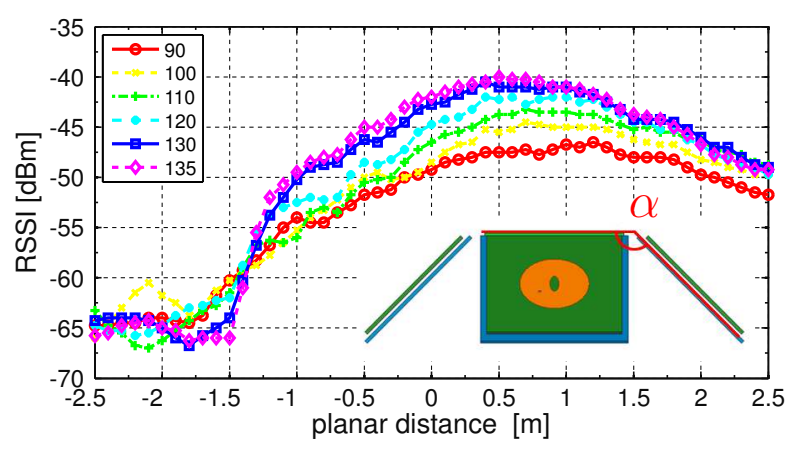

Fig. 6: Simulated RSSI distribution profile for various $\alpha$.

As the dihedral angle vary from the extremes $90^{\circ}$ to $140^{\circ}$, the power distribution changes accordingly, as depicted in Fig. 6. The position of the RSSI maximum under the SBA slowly shifts inward with increasing $\alpha$, while the peak value decreases. This phenomenon has consequences on the expected precision of the localization algorithm.

\section{B. Montecarlo simulations}

On the basis of the previous considerations, a montecarlo simulation is presented. With the generated data, a set of
RSSI repetition affected by noise were were modeled. The number of considered sample is only ten, low as in a real-time experiment, and $\sigma=1 \ldots 5$ were considered for the gaussian noise standard deviation. Canonical MUSIC localization is executed for each case, and in the following error statistics are presented.

Fig. 7 shows the distribution of the error as the dihedral angle vary from $90^{\circ}$ to $130^{\circ}$. The central region of each examined area is the most accurate in every case. This was expected, since this point has the best combination of data information. The error tend to shows a circular symmetry, as a consequence the uniform shape of the SBA.
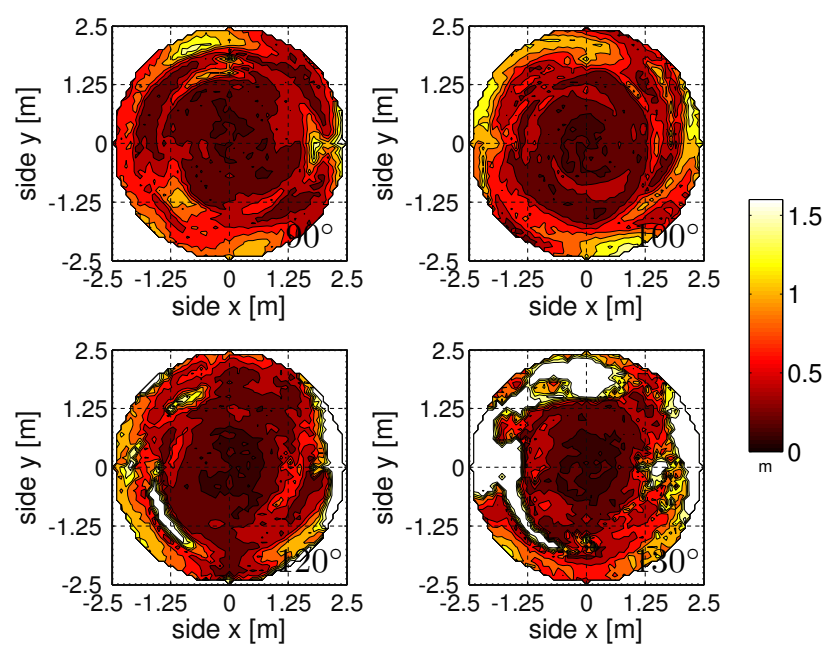

Fig. 7: Distribution of localization error for 4 dihedral angles.

Fig. 8 shows the cumulative distribution of the error for considered dihedral angles, in the case of $\sigma=1,2.5,5$. In the first case, (Fig. 8a) the traces shows a cross-over around $35 \mathrm{~cm}$ : below this values the best configurations are $\alpha=130^{\circ} / 14^{\circ}$, which offer a better coverage. After the cross-over the case between $90^{\circ} / 100^{\circ}$ performance better. When $\sigma$ rises, as in Fig. $8 \mathrm{~b}$ the global error rises, making the coverage smaller, and the relationship between the coverage changes. In particular, the $\alpha=110^{\circ} / 120^{\circ}$ are the best below error of $60 \mathrm{~cm}$, then $90^{\circ}$ is again the best configuration. As the noise became very severe, as the case of $\sigma=5, \alpha=90^{\circ}$ outperforms the set.

A more interesting information is obtained inspecting the plots of Fig. 9, which show the radial error density, the mean error for progressive circular crown. Focusing on the case of $\sigma=1$, the region delimited by $\rho<125 \mathrm{~cm}$ is better covered with high dihedral angle - in particular $\alpha=130^{\circ}$ seems the best. In turns $\alpha=90^{\circ} / 110^{\circ}$ are almost equivalent in the region $1.25<\rho<2.5$.

For $\sigma=2.5$, depicted in Fig. 9b, the performance partition is different: $\alpha=110^{\circ}$ is the best condition for $\rho<1 \mathrm{~m}$, but $\alpha=90^{\circ}$ is still the best arrangement for the outer area.

Finally, when the noise is extreme $(\sigma=5)$, the solution $\alpha=90$ is the best, even if the only metrically accurate. 


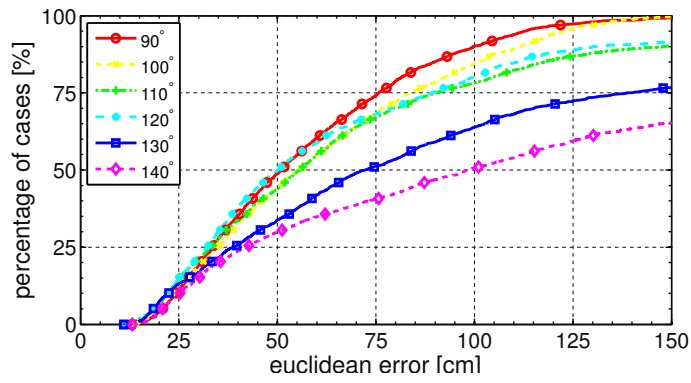

(a) $\sigma=1.0 \mathrm{~dB}$

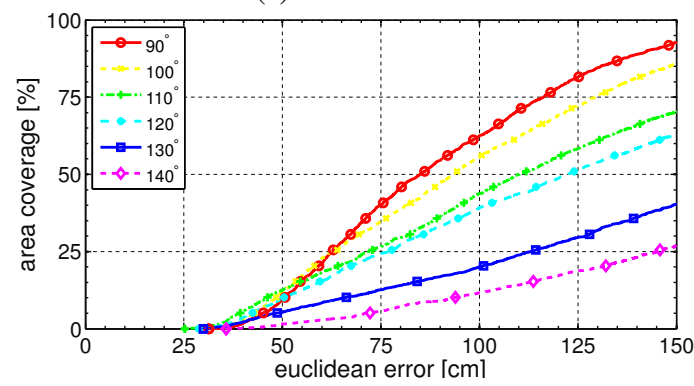

(b) $\sigma=2.5 \mathrm{~dB}$

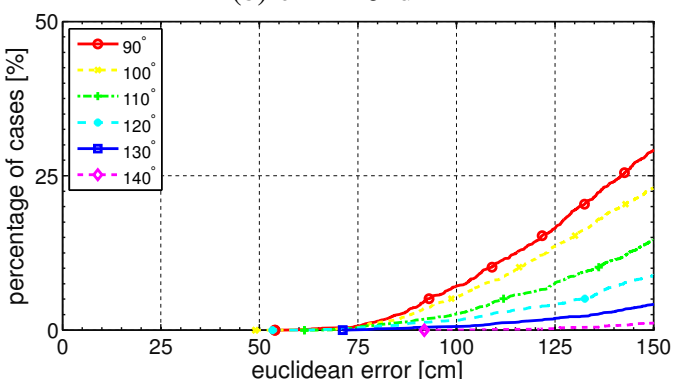

(c) $\sigma=5.0 \mathrm{~dB}$

Fig. 8: Error distribution for the six cases of dihedral angle.

\section{Conclusions}

An investigation about the impact of Switched Beam Antenna topology for indoor positioning system was presented. Based on the results of an accurate electromagnetic model, the investigation translates in a guideline for the class of positioning system based on RSSI measurement. In particular, varying the dihedral angle of cubic-like SBA, the distribution of the radial error density changes, impacting on the potential performance of a network of nodes. The arrangement of the SBA has to be chosen according to system requirements of higher level. In a noisy ambiance a wide coverage, while coarse, is reached for low dihedral angle. In a more controlled situation, an higher angle in the range $110^{\circ} / 130^{\circ}$ grants better local performance, hence narrower area of operation. In this case, to grant a global level of accuracy, a dense set of anchors must be considered.

\section{REFERENCES}

[1] A. Boukerche, H. Oliveira, E. F. Nakamura, and A. A. Loureiro, "Localization systems for wireless sensor networks," wireless Communications, IEEE, vol. 14, no. 6, pp. 6-12, 2007.

[2] S. Maddio, A. Cidronali, and G. Manes, "Smart antennas for directionof-arrival indoor positioning applications," in Handbook of Position Location: Theory, Practice, and Advances. Wiley Online Library, 2011, pp. 319-355.

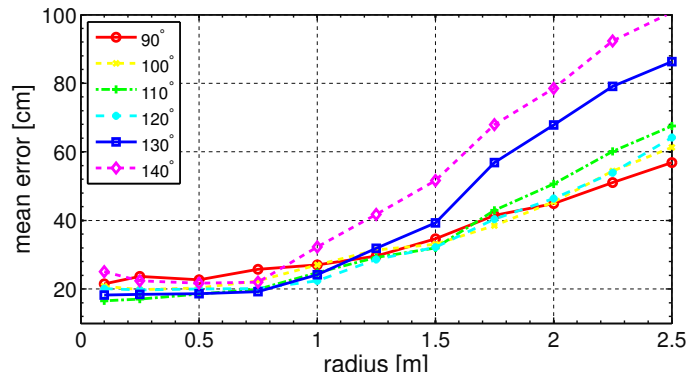

(a) $\sigma=1.0 \mathrm{~dB}$

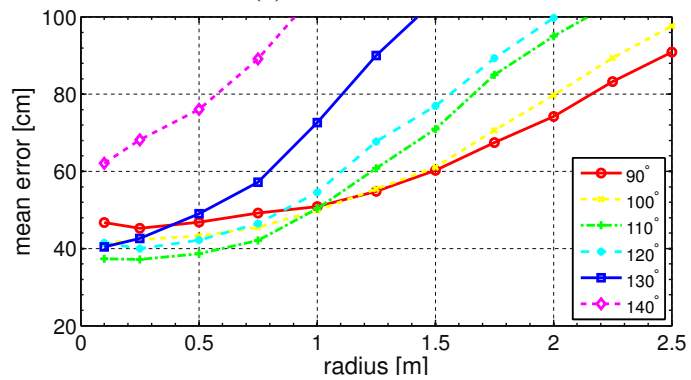

(b) $\sigma=2.5 \mathrm{~dB}$

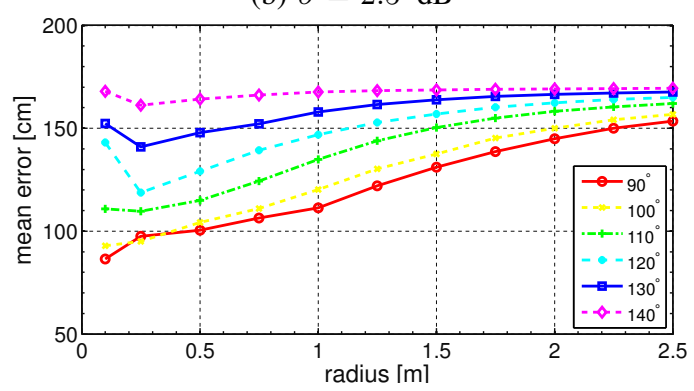

(c) $\sigma=5.0 \mathrm{~dB}$

Fig. 9: Error density for the six cases of dihedral angle.

[3] S. Maddio, M. Passafiume, A. Cidronali, and G. Manes, "A scalable distributed positioning system augmenting wifi technology," in Indoor Positioning and Indoor Navigation (IPIN), 2013 International Conference on. IEEE, 2013, pp. 1-10.

[4] R. Szumny, K. Kurek, and J. Modelski, "Attenuation of multipath components using directional antennas and circular polarization for indoor wireless positioning systems," in Radar Conference, 2007. EuRAD 2007. European. IEEE, 2007, pp. 401-404.

[5] R. Garg, Microstrip Antenna Design Handbook. Artech House, 2001.

[6] S. Maddio, A. Cidronali, and G. Manes, "A new design method for single-feed circular polarization microstrip antenna with an arbitrary impedance matching condition," IEEE Transactions on Antennas and Propagation, vol. 59, no. 2, pp. 379-389, 2011.

[7] A. Cidronali, S. Maddio, G. Giorgetti, and G. Manes, "Analysis and performance of a smart antenna for 2.45-ghz single-anchor indoor positioning," Microwave Theory and Techniques, IEEE Transactions on, vol. 58 , no. 1 , pp. 21-31, 2010.

[8] R. Schmidt, "Multiple emitter location and signal parameter estimation," Antennas and Propagation, IEEE Transactions on, vol. 34, no. 3, pp. 276-280, 1986.

[9] H. Krim and M. Viberg, "Two decades of array signal processing research: the parametric approach," Signal Processing Magazine, IEEE, vol. 13, no. 4, pp. 67-94, 1996.

[10] G. Giorgetti, S. Maddio, A. Cidronali, S. Gupta, and G. Manes, "Switched beam antenna design principles for angle of arrival estimation," in Wireless Technology Conference, 2009. EuWIT 2009. European. IEEE, 2009, pp. 5-8.

[11] Y. Chen, J. Yang, W. Trappe, and R. P. Martin, "Impact of anchor placement and anchor selection on localization accuracy," in Handbook of Position Location: Theory, Practice, and Advances. Wiley Online Library, 2011, pp. 425-455. 